\title{
Sedation failures in children undergoing MRI and CT: is temperament a factor?
}

\author{
TERRI VOEPEL-LEWIS RN, MSN*, SHOBHA MALVIYA MD*, \\ GERRIE PROCHASKA RN, BSN† AND ALAN R. TAIT PhD* \\ Departments of *Anesthesiology and + Radiology, Section of Pediatrics, \\ University of Michigan Health Systems, Ann Arbor, MI 48109, USA
}

\begin{abstract}
Summary
This study examined the relationship between temperament and sedation failure during magnetic resonance imaging (MRI) and computerized tomography (CT). One hundred and two children (aged 3-7 years) who underwent MRI or CT with or without sedation were studied. Demographics, sedatives administered, efficacy of sedation, and adverse events were recorded. Parents completed the Behavioural Style Questionnaire for 3-7 years olds during their wait. Eight children underwent MRI successfully without sedation, 83 were successfully sedated, and 11 procedures were aborted. Children whose sedation failed were less adaptable than children whose sedation was successful $(P=0.04)$. Children who underwent MRI without sedation were more persistent than children who were sedated $(P=0.05)$, and more persistent and less active than those whose sedation failed $(P=0.02$ and 0.03 , respectively). The child's underlying temperament may contribute to sedation outcomes during MRI and CT. A presedation assessment of the child's temperament may therefore assist in decisions regarding sedation.
\end{abstract}

Keywords: paediatric sedation; temperament; sedation failure

\section{Introduction}

Children frequently require sedation in order to be cooperative and immobile for magnetic resonance imaging (MRI) and computerized tomography (CT) procedures. As a result of increased emphasis on safe sedation practices and recommendations from the American Academy of Pediatrics (1), anaesthesiologists are frequently consulted regarding sedation issues for such procedures. Although a

Correspondence to: Terri Voepel-Lewis, Department of Anaesthesiology, F3900/Box 0211, 1500 East Medical Center Drive, Ann Arbor, MI 48109-0211, USA. variety of sedative agents, including chloral hydrate, benzodiazepines and pentobarbital, have been effectively used to facilitate these procedures, these agents fail to produce the depth of sedation necessary to complete the diagnostic test in $5-15 \%$ of cases (2, 3). Sedation failure is a concern not merely because of added costs to the institution, but because of inconveniences posed to the child and family, and the potential for delayed diagnoses. Indeed, sedation failure often necessitates an anaesthesia referral so that the diagnostic procedure may be facilitated with a general anaesthetic.

Unfortunately, it remains difficult to predict whether sedation is likely to succeed or fail for any 
given child. Previous studies have suggested that older children, those who have received either benzodiazepines as sole agents or combinations of agents, and those with a higher American Society of Anaesthesiologists (ASA) physical status, are at greater risk for sedation failure during diagnostic procedures $(2,4)$. Another study suggested that certain characteristics of the child's temperament may contribute to behaviour during sedation for dental procedures (5). To date, however, there are no published data regarding the relationship between temperament and sedation failure for diagnostic procedures. The purpose of this study therefore was to examine the relationship between temperament and sedation failure in children undergoing MRI and CT procedures. This study also evaluated the temperament of children who were able to undergo MRI without sedation.

\section{Methods}

With approval from the University of Michigan Institutional Review Board and parental consent, healthy children (ASA Physical Status I-II) aged 3-7 years scheduled to undergo MRI or CT procedures with sedation as outpatients were recruited. Children who were moderately to severely cognitively impaired were excluded. During the preprocedure assessment, the Behavioural Style Questionnaire (BSQ) for 3-7 years olds (6) was given to the parent(s) for them to complete while the child was in the scanner. This 100-item instrument is a valid and reliable tool that, when scored, produces numerical values (1, low/positive to 6 , high/ negative) for nine dimensions of temperament (Table 1). Temperament scores for each of these categories are entered onto a scoring grid that compares the child's individual scores to preestablished norms [means and standard deviations (SD)]. Scores within one SD from the mean describe the 'average' child, whereas scores <1 SD are positive, and those $>1$ SD are negative. In addition, the tool contains 10 questions related to the parent's overall perception of the child's temperament with regard to each of the nine categories and their perception of the child's overall manageability.

During the diagnostic procedure, the nurse caring for the child recorded medications administered, depth of sedation and adverse events including
Table 1

Definitions of Behavioural Style Questionnaire categories

\begin{tabular}{|c|c|}
\hline Adaptability & $\begin{array}{l}\text { Relative ease or difficulty in } \\
\text { responding to new situations }\end{array}$ \\
\hline Approach/withdrawal & $\begin{array}{l}\text { Nature of initial response to new } \\
\text { situations and people }\end{array}$ \\
\hline Threshold (sensitivity) & $\begin{array}{l}\text { Amount of stimulus necessary to } \\
\text { evoke a response from the child }\end{array}$ \\
\hline Intensity & $\begin{array}{l}\text { Energy level of the child's response, } \\
\text { irrespective of its quality }\end{array}$ \\
\hline Activity level & $\begin{array}{l}\text { Motor component of the child's } \\
\text { functioning }\end{array}$ \\
\hline $\begin{array}{l}\text { Rhythmicity/ } \\
\text { predictability }\end{array}$ & $\begin{array}{l}\text { Predictability of the child's } \\
\text { functioning (i.e. sleep, hunger) }\end{array}$ \\
\hline $\begin{array}{l}\text { Persistence (attention } \\
\text { span) }\end{array}$ & $\begin{array}{l}\text { Ability to concentrate over time; } \\
\text { capacity to continue despite obstacles }\end{array}$ \\
\hline Mood & $\begin{array}{l}\text { Amount of pleasant, friendly } \\
\text { behaviour compared to unpleasant } \\
\text { behaviour }\end{array}$ \\
\hline Distractability & $\begin{array}{l}\text { Degree to which extraneous stimuli } \\
\text { interferes with ongoing behaviour }\end{array}$ \\
\hline
\end{tabular}

inadequate sedation and sedation failures. Inadequate sedation was defined as difficulty completing the procedure due to the child's anxiety or inability to remain motionless. Sedation failures included procedures that had to be aborted due to inadequate sedation or a paradoxical reaction. In each of these cases, cancellation of the procedure was at the discretion of the nurse and attending radiologist after initial doses of a sedative agent and / or the addition of a second agent failed to produce a sufficient depth of sedation to complete the test. Aborted procedures were rescheduled to be completed either with a different sedative agent, or with a general anaesthetic.

During the study period, a small group of children in the same age range (i.e. 3-7 years) who underwent a successful MRI scan without any sedation were also studied. The decision to proceed with the MRI scan without sedation was subjectively made by the nurse during her preprocedure assessment of the child, and interview of the parent. In each of these cases, the child was deemed cooperative and attentive by the parent, and interacted well with the nurse. These parents were asked to complete the BSQ during the child's MRI procedure.

Temperament scores are presented as means \pm SD, and were analysed using unpaired $t$-tests. Nonparametric data including temperament categories, gender and medications were analysed 
Table 2

Description of the study groups

\begin{tabular}{llll}
\hline & Adequate sedation $(\mathrm{n}=83)$ & Failed sedation $(\mathrm{n}=10)$ & Not sedated $(\mathrm{n}=8)$ \\
\hline Age (years) & $4.7 \pm 1.4$ & $4.9 \pm 1.6$ & $5.6 \pm 0.9$ \\
Range (years) & $3-7.9(\mathrm{IQR} 1.75)$ & $3-7.5(\mathrm{IQR} 2.5)$ & $4-7(\mathrm{IQR} 1.0)$ \\
Gender (\%M/F) & $55 / 45$ & $50 / 50$ & $38 / 63$ \\
ASA status (\%I/II) & $76 / 24$ & $67 / 33$ & Not reported \\
Medications & & $7(70 \%)$ & Not applicable \\
$\quad$ Chloral hydrate (CH) & $68(82 \%)$ & $2(20 \%)$ & \\
CH +midazolam & $12(14 \%)$ & $1(10 \%)$ & \\
Midazolam & $3(4 \%)$ & & \\
\hline
\end{tabular}

IQR, interquartile range.

using chi-squared with Fisher's exact tests where appropriate. Pearson's product moment correlations were calculated to examine relationships between temperament scores and parental perceptions of behaviour. $P<0.05$ was considered significant.

\section{Results}

One hundred and 24 patients were asked to participate in the study over an 8-month period. Eight parents refused participation and 14 did not complete the BSQ. Therefore, the data presented were obtained from 94 children $(4.7 \pm 1.5$ years of age) who underwent MRI or CT with sedation and eight children (5.6 \pm 0.9 years) who underwent MRI without sedation. There were 11 aborted procedures, including 10 due to sedation failures, and one due to an allergic reaction and subsequent oxygen desaturation. For the purposes of this study, the child whose procedure was aborted due to an allergic reaction was excluded. All children in the nonsedated group underwent a successful MRI procedure. There were no significant differences in demographics between the children who underwent a successful procedure, those whose sedation failed, and those who required no sedation for their procedure (Table 2).

The mean temperament scores for each of the study groups are presented in Table 3 . Children with sedation failures were significantly less adaptable than children with successful sedation experiences $(P=0.05)$. Additionally, although not statistically significant, there were no 'positive' children in the sedation failure group (Figure $1 ; P=0.09$ ). The parents' general perceptions of the child's temperament correlated well with the temperament scores (Table 4); however, there were no significant relationships between parent global perceptions and sedation failure.

Children who underwent MRI without sedation were more persistent than children who were sedated $(P=0.05)$, and more persistent and less active than those whose sedation failed $(P=0.02$ and 0.03 , respectively). Additionally, $100 \%$ of children in this subset were classified as easy to manage by their parents, compared to only $60 \%$ of children in the failure group $(P=0.04)$.

\section{Discussion}

Sedation failures in children are frustrating for health care providers, as well as families, since failure generally ends in an aborted procedure, necessitating a return visit for the procedure to be completed with a different sedative agent or a general anaesthetic. The individual response to sedative agents remains variable, such that it remains difficult to predict with any certainty those children who may experience a failed sedation. Our findings suggest that the child's underlying temperament may contribute to sedation failure. Specifically, these data demonstrated that children with sedation failures were less adaptable than children with a successful sedation experience.

Temperament describes the inherent characteristics of a person that are relatively stable over time, and that predispose the individual to a particular behavioural style $(6,7)$. Nine categories of temperament have been studied extensively (Table 1) and, from these, investigators have clustered traits to describe the difficult child (i.e. arrhythmic, low in 
Table 3

Temperament scores of the study groups

\begin{tabular}{llll}
\hline & Adequate sedation $(\mathrm{n}=83)$ & Failed sedation $(\mathrm{n}=10)$ & Not sedated $(\mathrm{n}=8)$ \\
\hline Adaptability & $2.7 \pm 0.8$ & $3.3 \pm 1.1^{*}$ & $2.7 \pm 0.7$ \\
Approach/withdrawal & $2.9 \pm 0.9$ & $3.1 \pm 0.9$ & $3.1 \pm 0.9$ \\
Threshold (sensitivity) & $3.8 \pm 0.5$ & $3.6 \pm 0.3$ & $3.9 \pm 0.5$ \\
Intensity & $4.3 \pm 0.4$ & $4.4 \pm 0.4$ & $4.4 \pm 0.4$ \\
Activity level & $3.6 \pm 0.8$ & $4.0 \pm 0.5$ & $3.4 \pm 0.4^{* *}$ \\
Rhythmicity & $3.3 \pm 0.8$ & $3.5 \pm 0.7$ & $3.0 \pm 0.7$ \\
Persistence (attention span) & $3.2 \pm 0.7$ & $3.5 \pm 0.5$ & $2.7 \pm 0.7^{* * *}$ \\
Mood & $3.1 \pm 0.8$ & $3.5 \pm 0.6$ & $3.2 \pm 0.5$ \\
Distractability & $4.0 \pm 0.7$ & $3.8 \pm 0.6$ & $3.7 \pm 0.6$ \\
\hline
\end{tabular}

${ }^{*} P=0.04$ compared to adequate sedation group. ${ }^{* *} P=0.03$ compared to failed sedation group. ${ }^{* * *} P=0.05$ compared to sedated children; $P=0.02$ compared to failed sedation group.

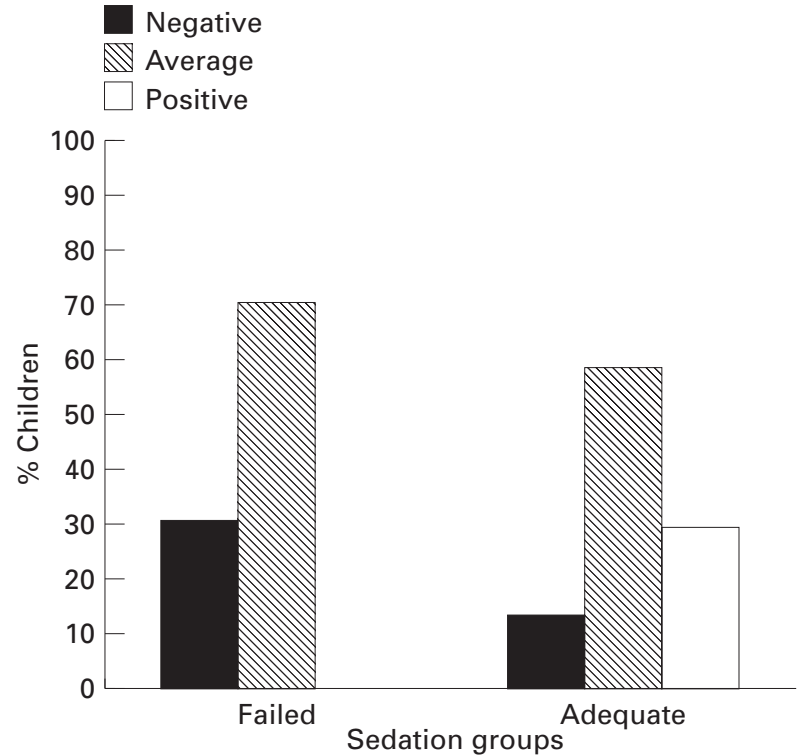

Figure 1

General mood of children in the study groups.

approach and adaptability, intense, predominantly negative in mood) and the easy child (i.e. rhythmic, adaptable, mild, and positive) (6). Using these theoretical constructs, several investigators have demonstrated a relationship between the child's temperament and his/her response to pain, hospitalization, and sedation (5,8-10). Wallace reported that children with a high level of intensity of responsiveness received more doses of pain medication during their hospital stay compared to other children (8). Schechter et al. found that children with 'difficult' temperaments exhibited more distress
Table 4

Correlations between temperament category scores and parent overall perceptions

\begin{tabular}{lc}
\hline & Correlation coefficients \\
\hline Adaptability & $0.628^{* *}$ \\
Approach/withdrawal & $0.622^{* *}$ \\
Threshold & $0.444^{* *}$ \\
Intensity & 0.024 \\
Activity level & $0.381^{* *}$ \\
Rhythmicity & 0.056 \\
Persistence & $0.643^{* *}$ \\
Mood & $0.676^{* *}$ \\
Distractability & -0.197 \\
\hline
\end{tabular}

**Correlation is significant at the 0.01 level (two-tailed).

symptoms during immunization than 'easy' children (9). These investigators additionally reported that adaptability had a strong negative correlation with high distress behaviour. Recently, Lochary et al. studied 29 consciously sedated toddlers undergoing dental restoration and found that temperament characteristics of approach/withdrawal and adaptability were predictive of struggling behaviour (5). Another study demonstrated that approachability and adaptability similarly predicted quiet behaviour during dental examinations in nonsedated 3-yearold children (11). Finally, Quinonez et al. reported that shyness predicted disruptive behaviour in nonpremedicated children undergoing general anaesthesia for dental procedures (12). Taken together, these studies suggest that the child's underlying temperament is associated with his/her behaviour during medical and dental experiences. 
Sedation is required to reduce anxiety and facilitate the immobility necessary to complete MRI and CT in many children, yet some are able to undergo these procedures without sedation. In our institution, nurses in the diagnostic areas assess the child's need for sedation based on initial contact and general interaction with the child, and the parent's perception of whether the child will require sedation for the procedure. During the study period, eight children were assessed as able to undergo MRI without sedation, and all procedures were successfully completed. These children tended to be more persistent (i.e. had greater attention spans) compared to children who required sedation, and were less active and more persistent than children who experienced failed sedation. Furthermore, all of these children were rated as easy to manage by their parents, compared to only $60 \%$ of those whose sedation failed. These data suggest that temperament is associated with the child's ability to undergo these diagnostic procedures without sedation.

In interpreting these results, it should be noted that although several elements of temperament were shown to differ significantly between the study groups, there may be other categories that would become significant in a larger sample. The possibility of a $\beta$ error for some of these temperament comparisons should not be ruled out. Furthermore, although the parents' global perceptions of the child's temperament were not associated with sedation failures in this sample, they did correlate well with temperament scores. Parental perceptions of elements of the child's behaviour may therefore be an important component of assessing the child's requirements for sedation, and his/her potential ease of sedating.

In conclusion, children whose sedation failed were less adaptable, and tended to be less positive in mood than children who were successfully sedated. Furthermore, children who underwent a successful procedure without sedation were less active, more persistent, and rated as easy to manage by their parents compared to children in the sedation failure group. Although further studies with a larger sample may be warranted, these findings suggest that the child's underlying temperament may contribute to sedation requirements and outcomes. A brief preprocedure assessment of the child's temperament with an emphasis on his/her ability to adapt to new situations, attention span and general mood, may be useful in predicting risk for sedation difficulties and, perhaps, in allocating sedation or anaesthesia resources.

\section{References}

1 Academy of Pediatrics Committee on Drugs. Guidelines for monitoring and management of pediatric patients during and after sedation for diagnostic and therapeutic procedures. Pediatrics 1992; 89: 1110-1115.

2 Malviya S, Voepel-Lewis T, Tait AR. Adverse events and risk factors associated with the sedation of children by nonanesthesiologists. Anesth Analg 1997; 85: 1207-1213.

3 Ronchera-Oms CL, Casillas C, Marti-Bonmati et al. Oral chloral hydrate provides effective and safe sedation in pediatric magnetic resonance imaging. J Clin Pharmacol Ther 1994; 19: 239-243.

4 Macarver-May DG, Kang J, Aouthmany M et al. Comparison of chloral hydrate and midazolam for sedation of neonates for neuroimaging studies. J Clin Pediatr 1996; 128: 573-576.

5 Lochary ME, Wilson S, Griffen AL et al. Temperament as a predictor of behavior for conscious sedation in dentistry. Pediatr Dent 1993; 15: 348-352.

6 McDevitt SC, Carey WB. The measurement of temperament in 3-7 year old children. J Child Psychiatry 1977; 19: 245-253.

7 McNeil TF, Persson-Blennow I. Stability of temperament characteristics in childhood. Am J Orthopsychiatry 1988; 58: 622-626.

8 Wallace MR. Temperament: a variable in children's pain management. Pediatr Nursing 1989; 15: 118-121.

9 Schechter NL, Berstein BA, Beck A et al. Individual differences in children's response to pain: role of temperament and parental characteristics. Pediatrics 1991; 87: 171-177.

10 Lee LW, White-Traut RC. The role of temperament in pediatric pain response. Issues Compr Pediatr Nurs 1996; 19: 49-63.

11 Radis FG, Wilson S, Griffen AL. Temperament as a predictor of behavior during initial dental examination in children. Pediatr Dent 1994; 16: 121-127.

12 Quinonez R, Santos RG, Boyar R et al. Temperament and trait anxiety as predictors of child behavior prior to general anesthesia for dental surgery. Pediatr Dent 1997; 19: 427-431.

Accepted 25 October 1999 